\title{
IMPACT SOCIO-ECONOMIQUE DE LA BAISSE DE LA PECHE LAGUNAIRE DANS LE DEPARTEMENT DE GRAND-BASSAM (CÔTE D'IVOIRE)
}

\author{
A.F.VANGA \\ Université de Bouaké /URES de Korhogo 27 BP 199 Abidjan 27, Côte d'Ivoire. Email : af_vanga@yahoo.fr
}

\begin{abstract}
RESUME
Dans la région de Grand-Bassam, la pêche en lagune a constitué l'activité économique principale des peuples Abouré Ehè et Ossouhon. Toutefois, une contrainte économique majeure a influencé la dynamique sociale du milieu. II s'agit de la fermeture de la communication entre la mer et la lagune à Grand-Bassam. Pour la collecte des données, les autorités coutumières et administratives, ainsi que les populations locales ont été enquêtées. Cela a consisté en des entretiens et observations. Les résultats des investigations ont montré que les différentes catégories sociales étaient ainsi impliquées dans la pêche en lagune. Cette activité économique a contribué au renforcement de la cohésion sociale et de la solidarité familiale. Depuis la fermeture de l'embouchure de Grand-Bassam dans les années 1950, il a été observé une baisse progressive de l'activité de pêche et de sa rentabilité économique. Chez les hommes, le revenu tiré de la pêche a chuté de $90 \%$ environ. Au niveau des femmes, cette baisse avoisine les $100 \%$. Cette situation a occasionné une reconversion économique des acteurs. Au plan social, la baisse de l'activité de pêche a entraîné une modification de la structure familiale. La disparition progressive de l'entraide familiale et l'émergence de ménages monoparentaux dirigés par des femmes, ont accentué la vulnérabilité des femmes et des enfants.
\end{abstract}

Mots clés : Impact, pêche, socio-économie, entraide familiale, Grand-Bassam, Côte d’Ivoire.

\begin{abstract}
SOCIO-ECONOMIC IMPACT OF THE FALL OF LAGOON FISHING IN THE DEPARTMENT OF GRAND-BASSAM (CÔTE D'IVOIRE)
\end{abstract}

In the Grand-Bassam area, lagoon fishing were the main economic activity of Aboure Ehe and Ossouhon communities. However, an external economic event influenced attitude of natives and had consequences on the social dynamic. It is about the closing of sea and lagoon communication at Grand-Bassam. Custom, administrative authorities and local populations were surveyed in order to collect informations. It consisted in interviews and observations. Results show that different social categories were involved in lagoon fishing. Also, economic activity contributed to the reinforcement of social stability and family solidarity. Since the closing of lagoon mouth in Grand-Bassam in 1950, involved a progressive fall of fishing and its economic profitability. The income of fishing of men fell off approximately $90 \%$. For women, the drop reached $100 \%$. This situation caused an economic mobility of actors. On the social level, a modification of social relationship. The disappearance of family mutual aid and the appearance of women head of the household, stressed the vulnerability of the women and children.

Keywords : Impact, fishing, socio-economy, family mutual aid, Grand-Bassam, Côte d'Ivoire. 


\section{INTRODUCTION}

L'abandon de certaines activités économiques jugées importantes au profit d'autres entraîne des bouleversements dont l'ampleur varie selon les sociétés. Dans le secteur de la pêche en Côte d'Ivoire, certains peuples riverains des cours d'eau avaient une profonde tradition halieutique (Anonyme, 2001 ; Berron, 1980 ; Verdeaux, 1994). Verdeaux (I.c.) a montré que les Aïzi, par exemple, avaient une tradition de pêche commerciale. La pêche qui constituait leur principale activité économique pendant la période précoloniale et coloniale, était liée à leur système social. L'abandon de cette activité par les neveux au profit de l'agriculture a entraîné une modification des rapports entre ces derniers et leurs oncles maternels dont ils dépendaient selon le système en question. Ainsi, une nouvelle dynamique sociale, induite par la mobilité économique, s'est imposée aux populations concernées. Le caractère progressif de ce changement n'a pas entraîné la destructuration systématique de l'ordre social existant. Al'instar du peuple Aïzi, la pêche lagunaire est restée longtemps une activité majeure qui a influencé la dynamique économique et sociale des Abouré Ehè et Ossouhon dans le Département de GrandBassam. En effet, la communication entre la mer et la lagune, à travers l'embouchure du fleuve Comoé à Grand-Bassam, a favorisé le développement de la pêche lagunaire.

Toutefois, la construction du canal de Vridi, sur la lagune Ebrié au niveau de la baie de PetitBassam à Abidjan, a entraîné une diminution progressive des chasses d'eau dans la communication entre la mer et la lagune à partir de 1952 (Moulod, 1995). II s'en est suivi une fermeture progressive de l'embouchure du fleuve Comoé dans la localité de Grand-Bassam. La conséquence directe de cette fermeture est le développement de végétaux aquatiques envahissants (Anonyme, 2004) et la disparition progressive de certaines ressources aquatiques (Albaret, 1994). Cette situation a entraîné une baisse de l'activité de pêche. Cela n'est pas sans impact sur le comportement économique et le système social du peuple Abouré qui vivait essentiellement de la pêche. L'objectif ici est d'analyser l'impact de l'activité de pêche sur la dynamique économique et sociale du peuple Abouré dans le Département de GrandBassam.
Cet article s'articule autour de deux grands points. Le premier analyse les caractéristiques socio-économiques liées à l'évolution de la pêche chez les deux sous-groupes Abouré considérés. Le second traite du contexte de la modification du système social des Abouré Ehè et Ossouhon.

\section{MATERIEL ET METHODES}

Cette étude est le résultat d'une enquête réalisée dans le Département de Grand-Bassam en 2004. Elle a porté sur deux des trois sousgroupes Abouré : les Akaba ou Ehè ${ }^{1}$ et les Ossouhon. Ces deux sous-groupes ont eu une tradition de pêche en lagune. Le troisième sousgroupe Abouré appelé Ehivè, qui s'est toujours consacré à l'agriculture, ne fait pas partie du champ de cette étude. Outre la revue documentaire, des guides d'entretien ont été utilisés comme support d'enquêtes. La collecte des données qualitatives a consisté en des entretiens individuels avec les autorités administratives et coutumières : le Préfet, le Sous-préfet, le Maire et la notabilité Abouré (4 personnes). Des entretiens de groupe ont eu lieu auprès des populations locales riveraines de la lagune Ebrié dans le Département de Grand-Bassam : 51 hommes et femmes ayant pratiqué la pêche au cours de ladite période, ainsi que 2 coopératives de jeunes. Les informations collectées portent sur la dynamique de l'activité de pêche lagunaire, les implications économiques et sociales de cette activité, et la situation socio-économique actuelle des Abouré Ehè et Ossouhon dans le Département de GrandBassam. Les informations obtenues lors des discussions de groupe ont été confrontées à celles des entretiens individuels. Dans le but de vérifier et de compléter les données, nous avons séjourné chez la population concernée afin de mieux observer les faits. Cela a permis d'obtenir, en plus des données qualitatives, des ordres de grandeur (Nombre de sorties de pêche, revenu moyen brut annuel des pêcheurs, proportion de la baisse dudit revenu, etc.).

Pour l'analyse des données, l'on a comparé l'activité de pêche ainsi que la situation économique et sociale des populations concernées avant et après la fermeture de l'embouchure de Grand-Bassam. En outre, la théorie du matérialisme historique a été requise

${ }^{1}$ Ce sous-groupe est ainsi « baptisé » par les autres sous-groupes Abouré. Cela signifie littéralement : ceux qui aiment des disputes (cf. Anonyme, 1998a) 
pour analyser la relation entre la baisse de la pêche lagunaire et la dynamique sociale.

\section{RESULTATS}

\section{CARACTERISTIQUES SOCIO-ECONOMIQUES LIEES A L'EVOLUTION DE LA PECHE CHEZ LES ABOURE EHE ET OSSOUHON}

\section{Tradition de pêche chez les Abouré Ehè et Ossouhon}

L'activité de pêche concerne le poisson et les crustacés. II ressort de l'enquête que six jours consécutifs sont consacrés à la pêche par semaine. Le dernier jour de la semaine est réservé aux cérémonies culturelles et cultuelles. En outre, les Abouré consacrent des jours à l'organisation de fêtes de réjouissance et de funérailles. Réservée aux hommes, la pêche au poisson et aux crustacées n'excède pas une période de 300 j dans l'année. Au cours de la période 1970 - 1980, la recette journalière en fonction de la période de pêche, était estimée en moyenne à $31000 \mathrm{~F}$ CFA pour le poisson, $11500 \mathrm{~F}$ CFA pour le crabe et à $13500 \mathrm{FCFA}$ pour la crevette. Ces données ont été obtenues en 2004 à partir de la confrontation des déclarations de notables, d'hommes et de femmes ayant pratiqué la pêche au cours de ladite période.

La pêche de crevette était une activité individuelle, tandis que celle de poisson et de crabe se pratiquait souvent de manière collective. En effet, les propriétaires de filets se faisaient aider soit par leur enfant ou le fils de leur sœur (neveu), soit par un frère, un cousin ou un ami. Dans les trois derniers cas, le propriétaire du matériel utilisé (filets et pirogue) répartissait le poisson pêché en trois : une part pour rémunérer sa participation à la pêche, une autre pour l'aide et la dernière part revenait au matériel utilisé (filets et pirogue). A travers ce mode de répartition du produit de la pêche, se manifeste le contrat social qui lie le détenteur des moyens de travail et ceux qui offrent leur force de travail. La part de poisson destinée au matériel de pêche représente la contrepartie en nature du moyen de production. Cette contrepartie revient, en fait, au propriétaire du matériel de pêche utilisé. Le fils et le neveu étant à la charge de ce dernier, aucune part du produit de la pêche ne leur est spécifiquement destinée. Ce système s'applique également à la pêche de crabe. Outre la pêche, il y avait le ramassage des coquillages et des huîtres, pratiqué par les femmes. La fréquence était de trois jours au maximum par semaine ; soit une estimation de 156 sorties dans l'année. Le ramassage se faisait par groupe de deux à trois femmes; le revenu tiré des produits de chaque sortie de ramassage était estimé à 9500 FCFA et réparti équitablement entre ces dernières. Le revenu annuel moyen brut d'une unité de pêche, calculé à partir de l'estimation des recettes journalières de 1970 à 1980, était de 9300000 FCFA pour les poissons, 4100000 FCFA pour les crevettes, 3500000 FCFA pour les crabes et de 1500000 FCFA pour les coquillages et les huîtres. La capture des poissons était l'activité qui procurait le plus de revenu. La régularité (environ 8 mois sur 12) et l'abondance des prises de bonne valeur marchande explique essentiellement cette rentabilité.

\section{Aperçu des activités et division sociale du travail}

Outre la pêche qui a constitué pendant longtemps la principale activité économique, les cultures ayant un intérêt économique sont le manioc, le café, le cacao, le cocotier, le palmier à huile et I'hévéa. Les deux dernières cultures sont d'introduction récente puisqu'elles remontent à 1975. En ce qui concerne les cultures de rente, les petites plantations de café et de cacao héritées de la colonisation, ne généraient pas de revenu satisfaisant. Les plantations de coco constituaient en quelque sorte des jardins d'ornement et leurs noix étaient vendues occasionnellement. Par ailleurs, les exploitations agricoles étaient de type familial. Elles étaient, de ce fait, gérées par des chefs de lignage. Le système social étant le système matrilinéaire, les enfants de la tante et ceux de la sœur faisaient également partie de la maind'œuvre familiale sur ces exploitations. Sur le plan foncier, les femmes n'avaient pas accès à la propriété de la terre. Toutefois, elles pouvaient accéder à des lopins de terre pour des cultures d'autosubsistance. Pour cela, ces dernières devaient en faire la demande auprès de leur chef de lignage. Lorsqu'il n'y avait pas de terre familiale disponible, la femme en faisait la demande auprès du chef d'un autre lignage par 
l'intermédiaire d'un adulte de sexe masculin, appartenant à son propre lignage. La production de manioc par les femmes, venait, pour la plupart, en appoint aux activités des hommes. Le manioc était généralement cultivé pour l'autoconsommation. Seul le surplus faisait l'objet de commercialisation. Cependant, des femmes (veuves ou célibataires), confrontées aux difficultés financières, commercialisaient, quelquefois, une partie de leur production. Les populations Abouré Ehè et Ossouhon n'étant pas agriculteurs de tradition, leur intérêt était orienté vers l'activité de pêche. Au niveau de cette activité, la classification, en ce qui concerne les catégories sociales, est plus nette comme l'indique le tableau 1. En fonction de la complexité de l'activité de pêche, cette classification découlait d'une organisation bien définie. En effet, dans le processus d'apprentissage, les jeunes commençaient par une activité moins complexe et moins pénible qui est la pêche des crabes. C'est une activité considérée comme moins valorisante et souvent exercée par un seul individu à l'aide d'un piège à crabe d'eau. Dans ce processus d'apprentissage, le jeune accompagnait l'adulte (le père ou l'oncle maternel) à la pêche des poissons. Cela se passait la nuit, ce qui permettait au jeune de se reposer le matin et de pratiquer la pêche de crabe l'après-midi. De retour au village, les jeunes scolarisés suivaient ce processus. En effet, contrairement aux jeunes non scolarisés et aux jeunes déscolarisés, ceux qui fréquentaient l'école exerçaient ces activités pendant les vacances scolaires. Cet apprentissage leur permettait de retourner à la pêche sans contrainte majeure en cas d'échec scolaire. Compte tenu de leur dextérité et de leur expérience, les adultes pratiquaient la pêche des poissons dans toute sa complexité. Ce type de pêche générait plus de revenus et suscitait, par conséquent, plus d'intérêt. La pêche des poissons était souvent pratiquée à deux. Quant à la prépondérance de la pêche de machoîrons (Chrysichthys sp.) et de crevettes (Macrobrachium sp.) chez les vieux, elle s'expliquait par le fait que ces derniers exerçaient une activité économique moins pénible physiquement, compte tenu de leur âge avancé. L'activité était menée à l'aide de bambou-pièges (pour les machoîrons) et de nasses en bambou (pour les crevettes) confectionnés généralement par les vieux euxmêmes. Cela occupait une partie non négligeable de leur temps. Par ce type d'activité, la catégorie d'individus en question se considérait comme utile à la société en participant à la production des richesses. La pêche des machoîrons et des crevettes était pratiquée dans les zones peu profondes de la lagune, proches de la rive.

Pour ce qui est de la pratique de la pêche par les femmes avant la fermeture de l'embouchure de Grand-Bassam, il s'agissait en fait d'activités de ramassage. Les huîtres étaient collectées dans les zones sableuses de la lagune, tandis que les coquillages, eux, se trouvaient dans les zones boueuses. L'activité ne nécessitait pas d'effort particulier. La présence de ces ressources dans la lagune était conditionnée par un niveau de salinité relativement élevé de l'eau. En plus du ramassage des coquillages et des huîtres, les femmes commercialisaient les produits de pêche des hommes de leur famille. Une femme qui a vécu cette période de ramassage, s'est exprimée en ces termes : " l'activité de ramassage des huîtres et des coquillages m'a permis d'acquérir des biens qui sont devenus rares aujourd'hui. Je peux mourir en paix car, personne ne pourra dire à mes filles, après ma mort, que leur mère n'a rien laissé de bon à ses héritières ». En outre, la qualité des habits et des parures lors des cérémonies de réjouissance, justifiait la recherche par la femme d'une source de revenu monétaire.

\section{Baisse de la rentabilité de la pêche liée à la fermeture de l'embouchure de Grand-Bassam}

La fermeture de l'embouchure de GrandBassam, en 1955, a entraîné la disparition progressive de la salinité de la lagune. C'est à partir des années 1980 que les effets de cette fermeture ont commencé à être perceptibles. Les végétaux aquatiques envahissants se sont ainsi développés sur le plan d'eau lagunaire. Ces végétaux flottants (laitues ou jacinthes d'eau) ont rendu difficile et inefficace la pêche lagunaire. La raréfaction des prises qui en a découlé a entraîné une baisse du revenu des pêcheurs. Comme on le constate dans le tableau 2, la baisse la plus importante concerne la pêche des huîtres et des coquillages (100 \%). Les femmes sont directement concernées par cette situation puisque ce sont elles qui pratiquaient le ramassage des huîtres et des coquillages. Au niveau de la pêche des poissons, la baisse du revenu a, dans un premier temps, occasionné une intensification des activités pour maintenir le revenu à un niveau acceptable par les pêcheurs. 
Tableau 1 : Technique de pêche selon la catégorie sociale, le lieu et la ressource pêchée.

Technique of fishing according to the social category place and the fished resource.

\begin{tabular}{llll}
\hline Catégories sociales & Engins utilisés & Sélectivité des engins & Zones de pêche \\
\hline Hommes jeunes & piège à crabe & crabes & profonde \\
Hommes adultes & filet maillant & poissons en général & profonde et très profonde \\
Hommes vieux & $\begin{array}{l}\text { bambou-piège et nasse } \\
\text { en bambou }\end{array}$ & mâchoirons et crevettes & peu profonde et profonde \\
Femmes & panier en bambou & huîtres et coquillages & peu profonde et berge \\
\hline
\end{tabular}

Tableau 2 : Estimation du revenu moyen brut annuel (F CFA) des pêcheurs en 2004 et du pourcentage de baisse par rapport à la période de prospérité économique.

Estimate of annual average income (F CFA) for fishermen in 2004 and the percentage of fall in relation to the period of economic prosperity.

\begin{tabular}{lcccc}
\hline Type de pêche & Poissons & Crabes & Crevettes & Coquillages et Huîtres \\
\hline Revenu moyen annuel & 700000 & 500000 & 350000 & 0 \\
Pourcentage de la baisse (\%) & 92 & 86 & 91 & 100 \\
\hline
\end{tabular}

\section{Stratégie de substitution de la pêche}

\section{Chez les hommes}

La baisse du revenu et la réduction du temps de pêche suite au développement des végétaux aquatiques envahissants, vont amener les différents acteurs de la pêche à s'orienter progressivement vers d'autres activités jugées plus rentables. Ainsi, les hommes Abouré ont progressivement changé d'activité pour devenir des agriculteurs. L'hévéa et le palmier à huile ont été plantés sur les parcelles de terres acquises antérieurement par les femmes. Les cultures de rente ont été développées par les hommes au détriment des cultures d'autosubsistance. La pêche qui constituait l'activité économique principale est devenue une activité secondaire pour ceux des hommes qui disposent de terres cultivables. Cette activité est désormais exercée de manière régulière par les pêcheurs "Ewè » originaires du Togo. La reconversion économique a eu des répercussions sérieuses sur la gestion du foncier.

\section{Chez les femmes}

Les femmes qui exploitaient les terres disponibles pour des cultures vivrières et qui assuraient leur subsistance et celle de la famille, pour quelques-unes, ont été dépossédées de cette ressource. Elles se sont, pour la plupart, reconverties dans le commerce de poissons et de crabes. Malgré l'irrégularité des produits collectés, elles demeurent dans l'activité pour s'assurer un revenu minimum. D'autres femmes sont, quant à elles, devenues productrices artisanales d'huile de palme.

\section{Chez les jeunes}

Les jeunes se sont transformés, pour certains, en exploitants de palétuviers et arbres forestiers le long des rives. L'exploitation sert à la commercialisation de bois de chauffe et de charbon de bois. D'autres jeunes, par contre, ont migré vers les centres urbains, soit pour rechercher un mieux-être, soit pour bénéficier de l'aide de parents vivant en ville et continuer ainsi leur scolarité. 


\section{CONTEXTE DE LA MODIFICATION} DU SYSTEME SOCIAL DES ABOURES

\section{Système matrilinéaire et entraide dans la société Abouré jusqu'à la baisse de la production de pêche}

La société Abouré repose sur le système matrilinéaire et le mode de production des richesses favorisait une hiérarchisation des relations sociales. Avant 1980, le jeune pratiquait la pêche de crabes à titre personnel et participait à la pêche des poissons en qualité de fils et d'apprenant. II ne disposait pas d'instruments personnels de pêche et n'avait généralement pas accès aux moyens de production. Selon les informations collectées, en tant qu'adulte en pays Abouré, l'individu pratiquait la pêche aux poissons sous toutes ses formes. Désormais libéré de ses obligations envers son père (donner une partie de sa production, aider dans les travaux champêtres, etc.), il bénéficiait de l'aide de son épouse et, plus tard, pouvait cumuler sa production et celle de ses fils. Il était, cependant, tenu de répondre aux sollicitations de son oncle maternel qui est le chef du lignage, et d'alimenter le trésor de ce lignage. Ainsi, à mi-parcours de son cursus, il recevait de ses fils et il donnait à son oncle. Enfin, lorsqu'il héritait de son défunt oncle maternel et que ses fils avaient acquis leur autonomie, le même individu changeait de statut. II atteignait ce rang de chef de lignage à un âge souvent avancé et pouvait, de ce fait, être classé dans la catégorie des « vieux ». Cet individu recevait aides et prestations de ses neveux, mais désormais exempt de toute forme de contribution envers son groupe de résidence. Au niveau économique, l'enquête auprès du peuple Abouré montre que la pêche aux crevettes et au machoîron est la principale activité pratiquée à ce stade. Le nouveau statut de chef de lignage ne lui conférait pas que des droits, il avait des devoirs : subvenir aux besoins matériels des membres de la famille, surtout des femmes qui sont plus vulnérables. Selon un notable, " un chef de lignage est décédé parce qu'il n'a pas accédé aux requêtes formulées par sa cousine maternelle. En effet, suite à l'incapacité (ou au refus) du chef de la loger en famille, cette dernière, en pleurs, avait demandé un dortoir aux ancêtres ». Pour notre interlocuteur, « ces faits sont considérés par les ancêtres comme une atteinte grave à la cohésion de la famille. Leur mécontentement avait entraîné le décès du chef de lignage en question dans le village d'Ebrah ».
Par ailleurs, la prospérité économique a eu un impact positif sur l'organisation de la société Abouré. Elle a permis une certaine émancipation économique des femmes. Les hommes étant en général des pêcheurs, les terres disponibles étaient cédées à des femmes par les chefs de lignage, pour les cultures d'autosubsistance. Ces cultures permettaient de satisfaire les besoins alimentaires de la famille. La commercialisation d'une partie de la production assurait un revenu à ces femmes. En ce qui concerne les enfants, leur éducation de base était assurée par toute la communauté ; ce qui facilitait l'intégration sociale des membres de la communauté à chaque étape de leur évolution. Par ailleurs, la modernisation du cadre de vie à travers l'habitat moderne, l'électrification rurale et l'accès à l'eau potable, au cours de la même période (dans les années 1970), avaient été en grande partie à l'actif des populations locales. La richesse des chefs de lignage leur conférait une autorité sur les autres membres de la société. Les rapports sociaux étaient fondés sur l'entraide et les valeurs sociales telles que le mariage constituaient une étape déterminante dans le processus de socialisation des hommes. Les hommes mariés avaient plus de considération dans la société que ceux qui ne l'étaient pas. Selon les informations recueillies, au moins trois femmes sur quatre $(75 \%)$ accédaient au mariage. L'achat d'objets de valeur pour la dot et la cérémonie de réjouissance, lors des mariages, constituaient une occasion pour l'homme d'exposer sa richesse financière. Ces pratiques contribuaient à la valorisation des femmes et à l'honneur de leurs familles. Au niveau de la structure de la famille, les frères et sœurs, les cousines maternelles, les neveux et nièces, les petits enfants étaient pris en charge par le chef de famille. L'organisation sociale étant fondée sur le système matrilinéaire, le fils de la sœur (le neveu) occupait une place déterminante dans la société. L'entraide, qui était une manifestation de la solidarité lignagère, guidait la logique familiale et permettait de prendre en charge les orphelins et les veuves. Selon les informations recueillies, la veuve demeurait dans la famille du défunt époux avec ses enfants, soit pour bénéficier de moyens de subsistance, soit en qualité d'épouse de l'héritier du défunt. Dans le premier cas, la veuve avait la possibilité de se remarier et de rejoindre son nouveau domicile conjugal avec ses enfants. Le nouvel époux assurait la subsistance des orphelins. 


\section{Disparition de l'entraide familiale}

La solidarité, dans sa manifestation sous la forme d'entraide familiale, est l'une des valeurs de la société Abouré. Elle tend à disparaître. En effet, la famille prenait en compte le chef de famille, son (ou ses) épouse (s), ses enfants, ses frères et sœurs, ses neveux, nièces et ses petits enfants. L'organisation sociale, soustendue par la prospérité économique avant 1980, permettait de prendre en compte tous les membres de la famille. Aujourd'hui, les rapports sociaux ont évolué vers l'individualisme. En effet, l'assistance mutuelle a progressivement disparu au profit de l'entraide au sein des familles prises de manière isolée. La famille Abouré élargie, représentée par le lignage, n'existe que de nom. Elle a été remplacée dans les faits par la famille restreinte. En général, les neveux, les nièces ainsi que les cousins et cousines, ne sont plus pris en charge financièrement dans les familles. II a été observé lors de l'enquête que des personnes vivent souvent dans la même maison familiale, mais ne partagent pas le repas familial sans avoir, au préalable, apporté une contribution financière. Toutefois, il existe quelques rares cas de dépendance vis-à-vis des parents exerçant une activité génératrice de revenu en milieu urbain. Comme le soutient un notable du village d'Ebrah, « C'est grâce à mon fils travaillant en ville que je vis dans des conditions acceptables. C'est lui qui paye les frais de l'eau et de l'électricité que j'utilise ici au village. Et chaque mois, il me fait parvenir de l'argent et des produits alimentaires pour ma subsistance ». Ces charges mensuelles, estimées à $21 \%$ du salaire du fonctionnaire ivoirien, constituent un supplément des charges ménagères de la population urbaine. Ce salaire excède rarement 350000 F CFA.

\section{Situation matrimoniale}

Les enquêtes montrent l'existence de plus en plus des ménages monoparentaux dirigés par des femmes. En effet, selon les informations recueillies, deux femmes sur trois qui ont des enfants à charge sont, célibataires, divorcées ou veuves. Par ailleurs, le mariage coutumier qui constituait la forme achevée du processus de socialisation des membres de la société Abouré, a progressivement disparu au profit d'unions libres. En effet, les conjoints ne sont plus liés par des engagements familiaux que représentent le plus souvent la dot et la vie sous le même toit. La tendance actuelle est à la séparation de résidence des concubins. Bien qu'ayant une relation avec un homme, les femmes demeurent dans la maison familiale. L'homme espère ainsi se décharger sur sa bellefamille puisque son union avec la femme n'est pas officialisée.

C'est le cas d'un homme et une femme qui ont vécu ce genre de relation pendant quatre années. Au cours de la première année, la fillette qui est née de leur relation amoureuse est restée à la seule charge de la mère. Le père a justifié sa non-participation aux charges de l'enfant par ses difficultés financières. Pour aider sa petite sœur, un cadre de la Fonction Publique ivoirienne, lui a apporté régulièrement un soutien financier. $A$ la troisième année, la dame en question a accouché d'une deuxième fillette. Un an plus tard, le concubin de cette dernière a commencé à convoiter une autre femme. C'est ainsi qu'à la suite d'une dispute, ce dernier a décidé de rompre avec sa compagne, laissant à la charge de cette dernière leurs deux fillettes. Outre cette forme d'union, quelques hommes ont opté pour le célibat. Pour ces derniers, les difficultés d'accès à un revenu conséquent expliquent leur refus du mariage. Avec l'absence d'entraide, ils estiment ne pas être en mesure d'assurer les charges familiales en cas de mariage. "A partir du moment où je n'arrive pas à satisfaire mes besoins d'ordre matériel, je ne peux pas me surcharger avec une femme comme épouse ». Ces propos sont d'un ancien pêcheur du village de Moossou. Les femmes, quant à elles, sont de plus en plus seules (veuves et célibataires) avec des charges liées à leurs besoins et à ceux de leurs enfants.

\section{Situation des personnes vulnérables}

La pêche a contribué au développement local en permettant aux parents de scolariser tous leurs enfants. Les enfants avaient alors la possibilité de poursuivre leurs études, même à l'étranger, au moment où la pêche était économiquement rentable et où les parents avaient les moyens d'assurer leur scolarité. Aujourd'hui, avec la paupérisation des parents, certains enfants issus de ménages dirigés par un homme, se trouvent dans l'obligation d'interrompre leurs études par manque de moyens financiers adéquats. Comme le soutenait un vieil homme du village de Moossou, "Lorsque l'enfant arrive au Lycée ou à l'Université, les charges deviennent plus élevées. II faut devoir trouver un lieu où le loger et lui donner de l'argent pour subvenir à ses besoins 
quotidiens. Or, nous n'avons plus de revenus suffisants pour faire face aux charges familiales». Les jeunes démunis pratiquent, pour certains, la pêche de crabe, et pour les autres, le commerce du palétuvier comme bois de chauffe afin d'assurer leur scolarité et leurs fournitures. Au niveau des femmes, la situation est également préoccupante. Avec le statut de chef de ménage, elles sont seules à subvenir à tous les besoins de leurs enfants. Le cas d'un couple illustre cette contrainte. En effet, le couple est retourné dans le village d'Ebrah après une vingtaine d'années d'activité dans l'administration publique du pays. A leur arrivée, chacun des conjoints est allé vivre en famille, consacrant ainsi une séparation de fait. Les enfants ont été confiés à leur mère. Leur père, quant à lui, s'est progressivement désengagé des charges liées à ces enfants. Bien qu'ayant le statut de femme mariée, elle vivait comme chef de ménage. Elle assurait sa propre subsistance et celle de ses enfants, ainsi que la scolarité de ces derniers. Pendant un moment, cette dame a bénéficié, de l'assistance de sa grande sœur qui réside en milieu urbain. Par la suite, les enfants ont été exclus du système scolaire puisque la mère n'avait plus les moyens d'assurer leur scolarité et le père refusait d'assumer ses responsabilités. La dame a fini par demander le divorce pour tenter une aventure de concubinage avec un autre homme du village. Pour elle, cela répondait à un besoin d'amélioration de ses conditions d'existence. Dans le Département de GrandBassam, les filles mères, les femmes célibataires, divorcées et veuves, d'une manière générale, ne bénéficient souvent plus d'aide matérielle et financière de la part des parents. Elles sont souvent l'objet de discrimination quant à l'accès à certaines activités génératrices de revenu. Pour subvenir à leurs besoins matériels, ces femmes se livrent à des comportements sexuels à risque. L'observation des faits a permis de constater que les femmes en question entretiennent des relations amoureuses discrètes avec des hommes nantis. Comme conséquences, environ trois jeunes filles sur quatre ont au moins un enfant qui est issu de ce type de relation à risque. Les adolescentes sont plus exposées à des grossesses précoces et non désirées.

\section{DISCUSSION}

Les populations Abouré Ehè et Ossouhon ont une tradition de pêche commerciale. Cela s'explique par le fait que dans la migration du groupe Akan dans l'actuel Côte d'Ivoire (Perrot, 1982), ces populations n'ont pas voulu suivre leurs frères Ehivê dans leur localisation actuelle « au bord de la forêt » (du nom de leur localité qui est Bonoua). Ils ont continué leur chemin à la recherche d'un site plus favorable à la pratique de leur activité économique principale : la pêche (Anonyme, 1967). Ce qui les a conduit au bord de la lagune Ebrié. Les Abouré Ossouhon, insatisfaits du site de "Moossou » retenu par les Ehè, auraient voulu découvrir d'autres sites plus propices à la pêche ; ce qui les aurait conduit sur l'actuel site appelé «Ebrah» (Anonyme, 1998a).

Dans la société Abouré, le mode de production des richesses favorisait une hiérarchisation des relations au sein du système matrilinéaire. Chez les Aïzi, le produit du travail d'un homme était remis à son père, tant qu'il était en position de « fils ». Selon Verdeaux (1994), le cursus de l'individu de sexe masculin le faisait passer par les statuts de « fils », de "père-neveu " et d' « oncle ». Le « fils » acheminait une partie de ses biens chez son oncle maternel qui faisait office de chef de lignage. L'autre partie était conservée par le père comme trésor familial. Jusqu'à l'âge de 30 ans environ, l'individu donnait sans presque rien recevoir, si ce n'était pour satisfaire ses besoins de subsistance. Lorsqu'il atteignait la position de "père-neveu » aux environs de 30 ans, le même individu se trouvait à la tête d'une unité de production autonome.

Comme le souligne Verdeaux (I.c.), dans la société Aïzi, l'homme recevait mais ne redistribuait plus lorsque qu'il se trouvait en fin de cursus social. II gérait le patrimoine du lignage. A l'instar d'autres sociétés traditionnelles, il y a un lien entre le monde des vivants et les ancêtres dans la croyance des Abouré. Ces ancêtres veillent sur la cohésion du lignage par le contrôle des actes que pose le chef de lignage en sa qualité de gérant du patrimoine du lignage. Un adage Abouré dit : « les morts ne sont pas morts. Ils vivent sous une autre forme à nos côtés ». De ce fait, une mauvaise gestion des biens que les ancêtres ont légués, entraîne des représailles de leur part.

La pêche, en tant que tradition et activité économique, est liée à l'organisation sociale des populations Abouré concernées. Cette activité économique, exercée aussi bien par les hommes que les femmes, n'est pas le fait des seules 
femmes Abouré. Au Sénégal, par exemple, les femmes Diola participent aussi à l'activité de pêche en allant en pirogue ramasser les huîtres en marée basse (Cormier-Salem, 1990). Les huîtres de Casamance proviennent uniquement de la cueillette sous les palétuviers, effectuée par les femmes Diola. Elles participent à la commercialisation des huîtres quand il n'y a plus de poissons. Leurs revenus varient de 15000 à $140000 \mathrm{~F} \mathrm{CFA}$ à la fin de la saison. Outre la vente d'huître, ces femmes pêchent des alevins, transforment des produits de la brousse comme le néré ou les palmistes, la vannerie, la cotonnade, la poterie, le maraîchage, la plantation d'arbres fruitiers.

Dans le Département de Grand-Bassam, l'exercice d'activités génératrices de revenus chez les femmes s'explique en partie par l'importance accordée à l'héritage dans la société Abouré. Cela peut aussi s'expliquer par la précocité du contact des populations côtières avec le capitalisme européen. En effet, le contact avec les négociants débarquant sur les côtes à partir du $14^{\mathrm{e}}$ siècle, depuis les Portugais jusqu'aux Français en passant par les Danois et les Hollandais, paraît un élément non négligeable dans la culture mercantile acquise par cette population (Anonyme, 1967).

La viabilité de la pêche commerciale était liée à la communication entre mer et lagune au niveau de Grand-Bassam. Cela contribuait à l'augmentation de la richesse en ressources aquatiques et, surtout, à la proportion entre formes estuariennes et marines (Albaret et Ecoutin, 1991). Selon ces auteurs, la lagune Ebrié contenait $65 \%$ d'espèces marines avant la fermeture de l'embouchure de Grand-Bassam en 1955. Après la fermeture de cette communication, il y a eu un développement des végétaux aquatiques envahissants sur le plan d'eau lagunaire. En effet, les feuilles entremêlées et flottantes de cette ressource peuvent doubler de taille en moins de deux semaines, et par leur seule densité, rendre la pêche et la navigation quasi impossible (Anonyme, 2004). Le matériel de pêche est le plus souvent détruit ou emporté par les végétaux aquatiques. Par ailleurs, ces plantes et leurs débris absorbent l'oxygène de l'eau, augmentent son acidité et ralentissent son débit, entraînant ainsi l'accumulation des alluvions (Anonyme, I.c.).

Outre la pêche, ces contraintes écologiques ont entraîné la raréfaction progressive des ressources halieutiques. Un cas similaire s'est présenté au niveau du lac d'Ayamé (Vanga, 2001). Quant à la pêche des huîtres et des coquillages, son abandon progressif est dû au fait que la survie de ces ressources dans la lagune Ebrié est liée à la présence du sel dans ce plan d'eau. Or, la fermeture de la communication de la lagune Ebrié avec la mer, à partir du fleuve Comoé à Grand-Bassam, a entraîné la disparition de cette salinité et par conséquent, la disparition des ressources en question.

Aussi, a-t-on constaté une dégradation progressive de la situation économique et sociale des acteurs de la pêche. Pour lutter contre la pauvreté, les populations concernées ont tenté une reconversion économique. Selon Heidrich (2001), les hommes réagissent à leur pauvreté en diversifiant leurs activités. Al'instar des peuples Aïzi (Verdeaux, 1994), les Abouré ont laissé la pêche devenir la « propriété » des populations originaires des pays de la sous région. Au Bénin par exemple, face à l'insuffisance de revenus, les hommes ont pratiqué l'élevage de volaille, d'ovins, de caprins et surtout de porcins. Ils ont aussi exploité leur littoral en plantant des cocoteraies (Pliya, 1980).

Quant aux femmes, la plus grande partie de leur revenu, dans certaines zones de la Côte d'Ivoire, provient de la vente de poisson, de riz, de produits et d'activités extra pêche de saison creuse comme la production " d'attiéké», " d'attoukpou» (type d'attiéké ayant la forme d'un disque) et de charbon (De Plaen et Geneau, 2002). A l'instar de celles de Côte d'Ivoire, des femmes du Bénin, pour lutter contre la misère, préparent du coprah pour le compte des propriétaires des plantations de cocotiers. Elles utilisent I'huile de palme pour l'alimentation et pour la fabrication du savon. Elles vendent des noix de coco et les bourres. Elles fabriquent aussi du sel. L'argent obtenu sert à satisfaire les divers besoins de la maison (Pliya, 1980).

Toutefois, face à une reconversion économique difficile, l'exode rural est devenu pour la jeunesse Abouré, un moyen pour changer de condition de vie. Selon Verdeaux (1981), I'un des phénomènes majeurs de ces trente dernières années touchant les sociétés côtières est la migration des jeunes vers les villes et principalement Abidjan. La reconversion économique chez d'autres peuples lagunaires de la Côte d'Ivoire a été également évoquée par Verdeaux (1994). Les pêcheries lignagères ont été progressivement abandonnées au fur et à 
mesure que les plantations arbustives se sont généralisées. La marginalisation progressive de ces pêcheries a atteint sa phase finale lorsque les conditions de décollage puis de maintien de la nouvelle économie, désignée comme " économie de plantation " fondée sur les cultures du cacao et du café, ont été réunies. Cette reconversion est liée à des facteurs endogènes. C'est par la volonté des populations concernées, qu'une activité économique a disparu au profit d'une autre. Ce qui n'est pas le cas des Abouré de la Sous-préfecture de GrandBassam. En effet, au niveau de la lagune Ebrié, les populations de pêcheurs n'ont pu se reconvertir entièrement en agriculteurs à cause de leur tradition de pêche. Outre cette contrainte, la faible disponibilité de terres cultivables limite les possibilités d'extension des exploitations agricoles des Abouré de GrandBassam. Ces derniers connaissent désormais une paupérisation relative.

Aujourd'hui, les rapports sociaux ont évolué vers l'individualisme. Les raisons officielles évoquées sont les contraintes économiques, les phénomènes d'urbanisation et d'occidentalisation de nos sociétés en majorités rurales. Toutefois, la raison principale demeure la paupérisation de la société Abouré. Comme le montre la théorie du matérialisme historique, la base matérielle (infrastructure) conditionne le mode de penser (superstructure) de la société. Un changement dans les conditions de production des richesses d'une société entraîne de fait des changements dans la structure et le fonctionnement de ladite société. La famille étant le premier niveau de socialisation de l'individu, elle est conditionnée dans le cas présent par la tradition de pêche, génératrice de richesse des populations Abourés. Les contraintes liées à la production de cette richesse entraînent une reconfiguration de la structure et de l'entraide familiales. Plusieurs ménages monoparentaux sont ainsi créés au sein de chaque grande famille. II est de plus en plus question de " chacun pour soi " au sein de la structure familiale élargie. Cette situation ne concerne pas les cas de mariage et de décès où l'on constate encore une manifestation de l'entraide familiale. Même si la famille est maintenue au niveau biologique, les rapports sociaux ont été considérablement modifiés.

Contrairement au milieu urbain, la dispersion spatiale des ménages n'est pas perceptible immédiatement en milieu rural. En effet, les ménages ruraux cohabitent dans la maison de la famille élargie. Ils utilisent généralement le même espace, la même cuisine, le même salon et quelques fois les mêmes chambres à coucher. En milieu urbain, par contre, l'espace de chaque ménage est bien défini.

En ce qui concerne le mariage, ce n'est donc plus le seul acte fondateur du couple. L'articulation entre le couple et le mariage a changé et l'union libre est devenue un mode de vie pour ces populations. Dans un contexte de paupérisation relative du monde rural, cette stratégie permettrait à l'homme de ne pas s'engager dans toutes les charges concernant la femme et les enfants. Il est désormais question d'une union de fait et non une union de droit sous-tendue par sa matérialisation (le versement d'une dot) et le rapprochement de deux familles. Ainsi, les concubins ont la possibilité de mettre fin à leur relation de fait sans contrainte. En d'autres termes, le couple n'a plus pour fonction de maintenir le groupe, la religion, le portefeuille parental, la situation parentale. II sert, désormais, à établir un « contrat à durée déterminée » entre un homme et une femme qui se mettent en couple. Le jour où l'un des deux entrave le développement de l'autre, le contrat n'est plus respecté et le couple peut se séparer (De Lara, Internet). En France par exemple, les couples concernés par l'union libre sont passés de 450000 en 1975 à 2400000 en 1998 (Ferréol et al., 2002). Dans une société Abouré partagée entre tradition et modernité (voire occidentalisation), ce phénomène constitue une préoccupation majeure pour les autorités coutumières locales.

Par ailleurs avec les ménages monoparentaux dirigés par des femmes, les jeunes démunis sont quelquefois amenés à abandonner les études de manière précoce. Mitchell (2003) souligne ce fait en décrivant les contraintes auxquelles fait face l'enseignement dans les pays en développement : pauvreté, faim, absence d'accès à l'école, mauvaise santé, malnutrition. Comme le soutient les ménages dirigés par une femme seraient ainsi plus pauvres que les ménages dirigés par un homme, et cela en raison des multiples contraintes qui pèsent sur les femmes, en particulier les difficultés pour celles-ci d'accéder aux ressources productives. Dans certaines régions rurales d'Afrique, près de la moitié des familles est dirigée par des femmes (Anonyme, 1998b). Or, ces femmes n'ont qu'un contrôle restreint de leur revenu. Leur production sert de plus en 
plus à l'alimentation familiale, les privant ainsi de revenus monétaires propres qu'elles peuvent investir dans une activité économique.

La situation est identique dans des pays comme le Burkina Faso, le Sénégal et le Niger (CormierSalem, 1990 ; De Plaen et Geneau, 2002). Dans ces zones, les femmes vendent moins de $2 \%$ de leurs céréales. Le reste sert à l'alimentation familiale. A l'instar de la société Abouré, la condition de la femme constitue une préoccupation majeure dans la région du lac d'Ayamé (Vanga, 2004). Etant donné la tendance à la baisse de nombreuses économies en Côte d'Ivoire qui augmente le nombre de pauvres, les rapports avec les hommes (occasionnel ou officialisé par le mariage) peuvent constituer des occasions vitales pour ces femmes et ces jeunes filles d'obtenir la sécurité financière et sociale ou de satisfaire des aspirations matérielles. La combinaison de la dépendance et de la subordination précarise davantage les jeunes filles et les femmes. En effet, elles ne peuvent pas exiger des rapports sexuels protégés ou de mettre fin à des relations comportant des risques liés à la santé sexuelle et reproductive et au VIH/SIDA. Comme le révèlent Antoine et Nanitelamio (1990), la déscolarisation est propice à la fécondité des jeunes filles (15 à 19 ans). Selon les propos de madame la Ministre de la lutte contre le SIDA en Côte d'Ivoire, cités par Kodjo (2004), les femmes représentent aujourd'hui $57 \%$ des infections chez l'adulte et $75 \%$ des personnes infectées par le VIH SIDA chez les jeunes concernent les filles.

\section{CONCLUSION}

La viabilité de la pêche dans le Département de Grand-Bassam a longtemps consolidé la sécurité sociale, économique et affective au sein de la société Abouré. La cohésion de la famille, sous-tendue par des conditions économiques favorables, atténuait considérablement la vulnérabilité de la femme et de l'enfance.

Depuis plusieurs décennies, avec la fermeture de l'embouchure de Grand-Bassam, l'on a constaté une baisse du revenu issu de la pêche et une dégradation progressive de la situation économique et sociale des acteurs. La reconversion économique envisagée par la population ne s'est pas opérée entièrement. Cela a entraîné une paupérisation de cette population et une reconfiguration de la structure familiale et une disparition de l'entraide au sein de la famille élargie. En lieu et place de l'esprit de sacrifice et d'entraide qui existait dans le temps, un esprit de lutte pour la survie a pris place. Les rapports d'assistance n'existent presque plus. L'on a assisté à une prépondérance des ménages monoparentaux avec les femmes en tête. En outre, l'option de l'union libre a progressivement supplanté celle du mariage coutumier. Cette situation a pour conséquence une féminisation de la pauvreté ; ce qui accentue la vulnérabilité de la femme et de l'enfant.

\section{REFERENCES}

Albaret J. J. 1994. Les poissons, biologie et peuplements. In : J. R. Durand, P. Dufour, D. Guiral et S. G. F. Zabi (Eds.). Environnement et ressources aquatiques de Côte d'Ivoire. Tome II : les milieux lagunaires, Editions de l'ORSTOM, Paris : pp 239 - 279.

Albaret J. J. et J. M. Ecoutin. 1991. Communication mer-lagune : Impact d'une réouverture sur l'ichtyo faune de la lagune Ebrié. Arch. Scient. 1 (2) : 99 - 109.

Anonyme. 1967. Région du Sud-Est : Etude socioéconomique. La Sociologie. SEDES, Ministère du Plan, Paris, $131 \mathrm{p}$.

Anonyme. 1998a. Titre Monographique. Département de Grand-Bassam. Ministère de l'Intérieur et de la Décentralisation, Grand-Bassam, $16 \mathrm{p}$.

Anonyme. 1998b. Les femmes, que leur réserve l'avenir. Bimensuel "Réveillez-vous » $79(7): 3-10$.

Anonyme. 2001. Procès Verbal de Passation de service. Département de Grand-Bassam. Ministère de l'Intérieur et de la Décentralisation, Grand-Bassam, $16 \mathrm{p}$.

Anonyme. 2004. Coléoptères à la rescousse. Bimensuel « Réveillez-vous» 85 (9) : p 29.

Antoine P. et J. Naritelamio. 1990. La montée du célibat féminin dans les villes Africaines. Trois cas : Pikine, Abidjan et Brazzaville. Les dossiers du CEPED, Paris, $27 \mathrm{p}$.

Berron H. 1980. Tradition et modernisme en pays lagunaires de Basse Côte d'Ivoire. Editions OPHRYS, $369 \mathrm{p}$.

Cormier-Salem M. C. 1990. Une pratique revalorisée dans un système de production en crise : la cueillette des huîtres par les femmes diola de Basse Casamance 
(Sénégal). In : C. Aubertin et E. Bernus (Eds.). La pêche : enjeux de développement et objet de recherche. Edition de ORSTOM, Cah. Sc. Hum. 25 (1 - 2) : pp 91 - 106.

De Lara A. internet. Sociologie de la famille. F : IFamillelSocio Les familles monoparentales.htm

De Plaen R. et R. Geneau. 2002. Riziculture de bas-fond, autonomie des femmes et paludisme dans le Nord de la Côte d'Ivoire. In : G. Cahn (Ed.). Agriculture : Impact sanitaire et nutritionnel des hydro-aménagements en Afrique. Revue d'étude et de recherches francophones, 11 (1) : pp 17 - 21.

Ferréol G., P. Cauche, J. - M. Duprez, N. Gadrey et M. Simon. 2002. Sociologie de la famille. In : G. Ferréol, P. Cauche, J. - M. Duprez, N. Gadrey et M. Simon (Eds.). Dictionnaire de Sociologie. Armand Colin, $3^{\mathrm{e}}$ édition, Paris : pp 71 - 78.

Heidrich G. 2001. La communication pour des Moyens d'Existence Durable dans la Pêche (PMEDP). In : B. Horemans (Ed.). Programme pour des moyens d'existence durables dans la pêche en Afrique de l'Ouest. Bulletin PMEDP, Revue trimestrielle, Cotonou : pp 16 - 19.

Kodjo E. 2004. Lutte contre le SIDA. La féminisation de l'épidémie fait peser des menaces sur le socle social. Fraternité Matin $n^{\circ} 120204$. www. fratmat.net

Mitchell A. 2003. L'alimentation scolaire : Aujourd'hui plus que jamais. In : J. Albert
(Ed.). Alimentation, Nutrition et Agriculture, FAO, 33, Rome : pp 6 - 10.

Moulod J. M. 1995. Embouchure de GrandBassam : Impact socio-économique et environnemental. Conférence sur les enjeux de l'embouchure de GrandBassam, $14 \mathrm{p}$.

Perrot Cl. H. 1982. Les Anyi-Ndenyé et le pouvoir aux $18^{\mathrm{e}}$ et $19^{\mathrm{e}}$ siècles. CEDA, Abidjan, $48 \mathrm{p}$.

Pliya J. 1980. La pêche dans le Sud-ouest du Bénin. Agence de Coopération Culturelle et Technique, Paris, $278 \mathrm{p}$.

Vanga A. F. 2001. Conséquences socioéconomiques de la gestion des ressources naturelles: cas des pêcheries dans les lacs d'Ayamé et de Buyo (Côte d'lvoire). Thèse de Doctorat ès Sciences et Gestion de l'Environnement, Université d'Abidjan Abobo-Adjamé (Côte d'Ivoire), 210 p.

Vanga A. F. 2004. Conséquences socioéconomiques de l'expulsion des pêcheurs étrangers en Côte d'Ivoire : lacs d'Ayamé et de Buyo. Revue Européenne de Migrations Internationales, 20 (1) : 197 - 205.

Verdeaux F. 1981. L'Aïzi pluriel. Chronique d'une ethnie lagunaire de Côte d'Ivoire. Thèse de $3^{\mathrm{e}}$ Cycle, Ecole des Hautes Etudes en Sciences Sociales, Paris (France), $301 \mathrm{p}$.

Verdeaux F. 1994. Contexte sociologique. In : J. R. Durand, P. Dufour, D. Guiral, et S. G. F. Zabi (Eds.). Environnement et ressources aquatiques de Côte d'Ivoire. Tome II : les milieux lagunaires. Editions de l'ORSTOM, Paris : pp 367 - 398. 